\title{
Modulation of inflammatory mediators in the trigeminal ganglion by botulinum neurotoxin type $A$ : an organ culture study
}

\author{
Jacob Edvinsson ${ }^{1}$, Karin Warfvinge ${ }^{1,2^{*}}$ and Lars Edvinsson ${ }^{1}$
}

\begin{abstract}
Background: Onabotulinumtoxin type A (BoNT-A) has been found to reduce pain in chronic migraine. The aim of the present study was to ask if BoNT-A can interact directly on sensory mechanisms in the trigeminal ganglion (TG) using an organ culture method.

Methods: To induce inflammation, rat TGs were incubated for 24 hrs with either the mitogen MEK1/2 inhibitor U0126, BoNT-A or NaCl. After this the TGs were prepared for immunohistochemistry. Sections of the TG were then incubated with primary antibodies against CGRP (neuronal transmitter), iNOS (inflammatory marker), IL-1 $\beta$ (Interleukin 1ß), SNAP-25 (synaptic vesicle docking protein) or SV2-A (Botulinum toxin receptor element).

Results: We report that CGRP, iNOS, IL-1 $\beta$, SNAP-25 and SV2-A were observed in fresh TG with a differential distribution. Interestingly, NaCl organ culture of the TG resulted in enhanced expression of CGRP and SNAP-25 in neurons and iNOS in SGCs. Co-incubation with U0126 or BoNT-A retained the increased expression of SNAP-25, while it decreased the IL-1 $\beta$ immunoreactivity in neurons. The iNOS expression in SGCs returned to levels observed in fresh specimens. Moreover, we observed no alteration SV2-A expression in SGCs. Thus, the overall picture is that both U0126 and BoNT-A have the ability to modify the expression of certain molecules in the TG.

Conclusion: We hypothesize that chronic migraine might be associated with some degree of inflammation in the TG that could involve both neurons and SGCs. It is clinically well recognized that treatment with corticosteroids will reduce the symptoms of chronic migraine; however this remedy is associated with long-term side effects. Understanding the mechanisms involved in the expressional alterations may suggest novel ways to modify the changes and indicate novel therapeutics. The results of the present work illustrate one way by which BoNT-A may modify these expressional alterations.
\end{abstract}

Keywords: CGRP; iNOS; IL-1ß; SNAP-25; SV2-A; Monoclonal antibodies; Botulinum neurotoxin type A

\section{Background}

Migraine is a common neurological disorder that afflicts up to $16 \%$ of the adult population in the Western countries [1]. It is characterized by episodic, often disabling headache, associated with sensory, autonomic, central nervous system (CNS) related and cognitive symptoms. The current view is that migraine is a disorder in which CNS dysfunction plays a pivotal role while various parts of the trigeminal system are necessary for the expression of peripheral symptoms and aspects of pain [2].

\footnotetext{
* Correspondence: karin.warfvinge@med.lu.se

'Department of Medicine, Lund University, Lund, Sweden

${ }^{2}$ Department of Clinical Experimental Research, Glostrup Research Institute, Glostrup Hospital, Glostrup, Denmark
}

In a subgroup of migraine patients (1-2\%) the frequency of migraine may expand over time to multiple monthly attacks. Furthermore, these may progress to be chronic (attacks $>15$ days per month) and are often associated with medication overuse [3]. These patients are very difficult to treat. Onabotulinumtoxin type A (BoNT-A) has shown efficacy in the treatment of chronic migraine [4-6], however its mechanism of action remains in this relation elusive.

The effect of BoNT-A at the neuromuscular junction is well demonstrated [7]. The C-terminal of the toxin binds to the motor neuron and mediates endocytosis of the toxin [8]. Within the vesicle of the motor endplate it 
cleaves the vesicular docking protein SNAP-25, which leads to inhibition of acetylcholine storing vesicles docking on the presynaptic membrane and thus reduces acetylcholine release [8]. The potential of BoNT-A in treatment of migraine was suggested 15 years ago and observed in conjunction with cosmetic treatments [9]. Since then several suggestions to explain the antimigraine effect has appeared. The most obvious would be reduction in proprioceptive signaling to the brainstem but also decreased mechanical sensitivity of nociceptors and inhibition of craniofacial muscle tone [10-12]. These mechanisms were further developed and BoNT-A was suggested to interfere with expression of mechanosensitive ion channels on meningeal nociceptors [13].

The aim of the present study was designed to ask if BoNT-A can interact directly on sensory mechanisms in the trigeminal ganglion (TG) using an organ culture method [14-16]. With this method we can study whole TG, and the interrelation between neurons and satellite glial cells (SGC). The neuronal-glial signalling in the TG might be of much relevance in migraine and in particular in the chronification pathology that develops in many patients [17]. During organ culture there is an inflammation response elicited with increased expression of cytokines and mitogen-activated protein kinases (MAPK) [14-16]. We hypothesized that BoNTA might interfere with the expressional changes of the induced inflammation, and, in addition, the expression of SNAP-25 and the Botulinum toxin receptor element (SV2), molecules observed in the TG.

\section{Methods}

Ten Sprague Dawley rats (male, 200-250 g) were anesthetized with $\mathrm{CO}_{2}$ and decapitated. Right and left TG were removed and either used directly for experiments (fresh), or incubated in Dulbecco's modified Eagle's medium (DMEM; Gibco, Invitrogen, Carlsbad, CA, USA) supplemented with penicillin $\left(100 \mathrm{U} \mathrm{ml}^{-1}\right)$, streptomycin $\left(100 \mu \mathrm{g} \mathrm{mL}^{-1}\right)$ and amphotericin $\mathrm{B}\left(0.25 \mu \mathrm{g} \mathrm{mL}^{-1}\right)$ for 24 hours at $37{ }^{\circ} \mathrm{C}$ in humidified $5 \% \mathrm{CO}_{2}$ in air (for details on the method see Tajti et al.) [15]. Prior to start of the incubation the MEK1/2 inhibitor U0126 (LC laboratories, Boston, MA, USA) $10 \mu \mathrm{M}$, BoNT-A (3 units/mL, ALLERGAN) or an equal volume of $\mathrm{NaCl}$ (vehicle) was added. Incubation with the different substances was repeated 5-7 times. The experimental procedures were approved by the University Animal Ethics Commtttee (M43-07).

TG (either fresh or after 24 hours of incubation) were fixated in $4 \%$ paraformaldehyde (Sigma, St Louis, USA) in phosphate buffered saline (PBS) for 2-4 hours. After fixation TG were cryoprotected using $10 \%$ and $25 \%$ sucrose (Sigma) in Sorensen's phosphate buffer. Subsequently, the specimens were embedded in gelatin medium
(30\% egg albumin, $3 \%$ gelatin, Sigma), cryosectioned at $12 \mu \mathrm{m}$ and stored at $-20^{\circ} \mathrm{C}$ until use.

Sections were thawed in room temperature, then rehydrated in PBS containing $0.25 \%$ Triton X-100 (PBS-T; Sigma) for 15 minutes. Sections were incubated with primary antibodies in PBS-T, containing $1 \%$ bovine serum albumin (BSA; Sigma), overnight in $+4{ }^{\circ} \mathrm{C}$. After incubation with the primary antibody, sections were equilibrated to room temperature, rinsed in PBS-T for $2 \times 15 \mathrm{~min}$, followed by incubation with the secondary antibody for 1 hour in a dark room at room temperature (for details on antibodies, see Table 1). Sections were washed with PBST $2 \times 15 \mathrm{~min}$ and mounted with anti-fading mounting medium (Vectashield, Vector Laboratories, Burlingame, CA, USA). Omission of the primary antibody served as negative control. The immune-stainings were repeated 3-5 times. For general morphology evaluation, sections were stained in hematoxylin-eosin (Htx, Sigma).

Sections were examined and images were obtained using light- and epifluorescence microscope (Nikon 80i, Tokyo, Japan) equipped with a scanning stage for upright microscope (Märzhäuser, Germany) with travel range $\mathrm{X} / \mathrm{Y} 75 \times 50 \mathrm{~mm}$ automatic adjustment of the Zaxis, and coupled to a Nikon DS-2 MV camera. This enabled us to take large images of TG, with high resolution. Images were taken using NIS basic research software (Nikon, Japan). We estimated the number of immunoreactive cells and the intensity in these images; however, the detailed distribution was analyzed in regular images in $20 \times$ and $40 \times$ magnification. In addition, as a complement to the analysis, fluorescence intensity was measured in three sections of CGRP, IL-1 $\beta$ and SNAP25 stainings, since these showed neuronal immunoreactivity which could be measured. Mean intensity and SD were calculated. In iNOS and SV2-A stainings, the immunoreactivity was confined to the SGCs and could thereby not be used in fluorescence intensity measurements. Statistically, one-way ANOVA analysis and Bonferroni's multiple comparison test were used. $P<0.05$ was considered as statistically significant.

\section{Results}

\section{Morphology}

The morphology of the TG was evaluated following Htx staining (Fig. 1). Neurons of different size were

Table 1 Details on primary antibodies used for immunohistochemistry

\begin{tabular}{llll}
\hline Name & Host & Dilution & Supplier \\
\hline iNOS & Rabbit & $1: 200$ & Abcam; Cambridge, UK \\
IL1 $\beta$ & Rabbit & $1: 400$ & Abcam; Cambridge, UK \\
SNAP-25 & Rabbit & $1: 100$ & Sigma-Aldrich, St. Louis, MO, USA \\
SV2-A & Rabbit & $1: 1000$ & Abcam; Cambridge, UK \\
\hline
\end{tabular}




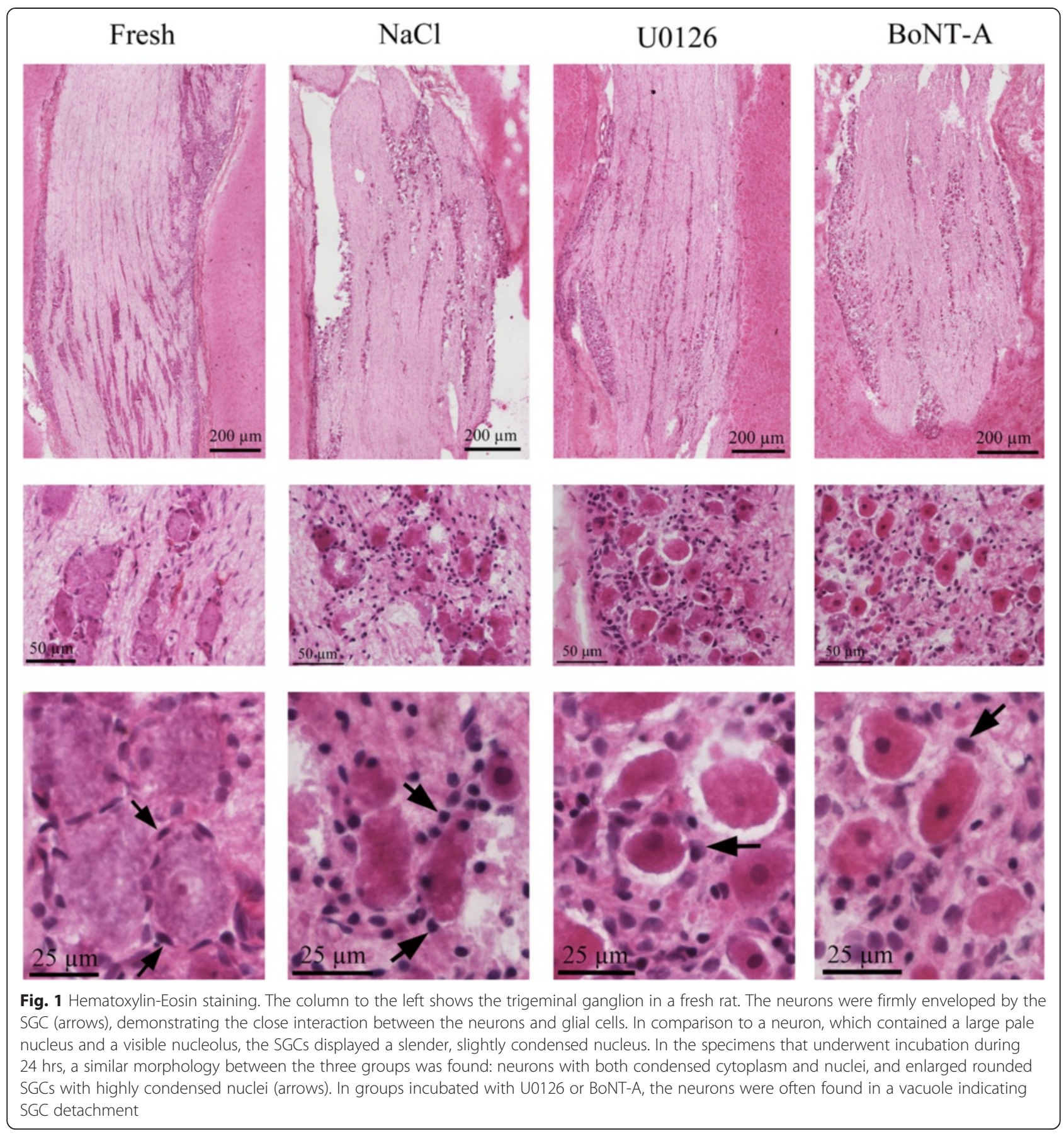

firmly enveloped by SGCs, demonstrating the close interaction between the neurons and the glial cells. The neurons contained a large pale nucleus and a visible nucleolus, and the SGCs displayed a slender, slightly condensed nucleus.

In the specimens that underwent incubation during 24 hours, a similar morphology between the three groups was observed: neurons with both condensed cytoplasm and nuclei, and enlarged rounded SGCs with highly condensed nuclei. Moreover, in groups treated with U0126 or BoNT-A, the neurons were often found in a vacuole indicating cell shrinkage and SGC detachment.

\section{Fresh TG}

In the fresh TG, we observed both CGRP positive and negative neurons (Fig. 2a). The CGRP immunoreactivity was confined to the cytoplasm in a granular pattern, resembling staining of the endoplasmatic reticulum. In 


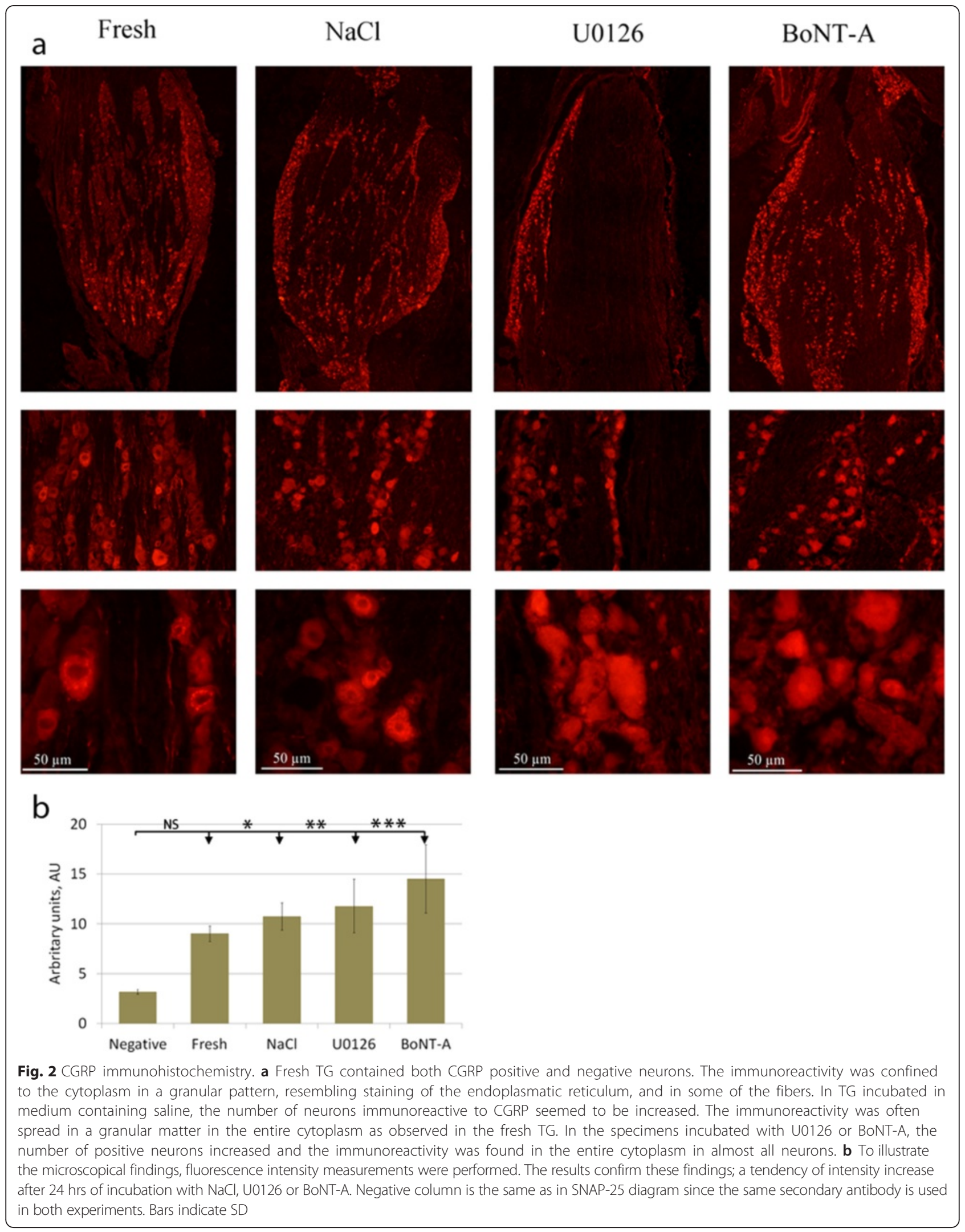


addition, some of the nerve fibers seen in TG were CGRP positive.

iNOS immunoreactivity was exclusively found in the SGCs (Fig. 3), and IL-1 $\beta$ immunoreactivity in almost all neurons, which appeared as granules in the cytoplasm (Fig. 4a).

Scattered cytoplasmatic SNAP-25 immunoreactive granules were found in most neurons (Fig. 5a) and SV2-A immunoreactivity in the cytoplasm of SGCs (Fig. 6).

\section{Vehicle incubation for $24 \mathrm{hrs}$}

In the $\mathrm{NaCl}$ incubated group (vehicle group), we observed no clear difference in CGRP immunoreactivity compared to fresh TG; both CGRP immunoreactive and negative neurons were observed (Fig. 2a). In addition, the granular intracytoplasmatic CGRP immunoreactivity found in fresh
TG was found after 24 hours of incubation with $\mathrm{NaCl}$. Immunoreactive CGRP positive fibers were also observed.

The SCGs displayed increased iNOS immunoreactivity compared to fresh TG (Fig. 3). As for the fresh specimens, almost all neurons in the incubated groups displayed IL-1 $\beta$ immunoreactivity, which appeared as granules in the cytoplasm (Fig. 4a).

Compared to the fresh specimens, there was increased SNAP-25 immunoreactivity in the neurons in the $\mathrm{NaCl}$ incubated group, to such an extent that it was difficult to discern the distribution of immunoreactivity within the neurons (Fig. 5a). We found no difference in SV2-A immunoreactivity between the fresh specimens and the incubation group (Fig. 6), except for the rounded shape of the SGCs appearing after 24 hours of incubation, as also was seen in the Htx staining (Fig. 1).

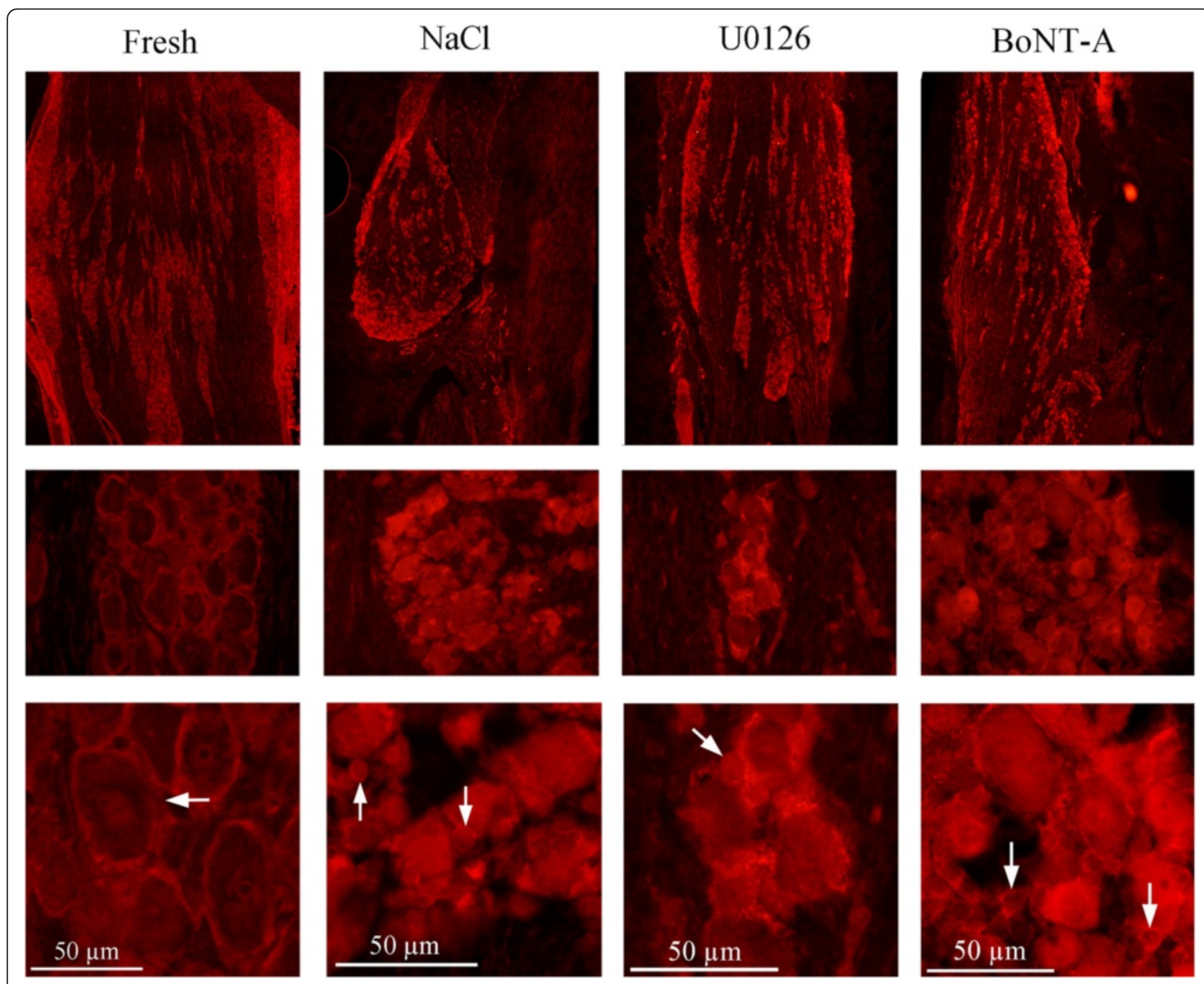

Fig. 3 iNOS immunohistochemistry. In fresh animals, the iNOS immunoreactivity was exclusively found in the SGCs (arrow). In the saline group, some neurons and neuronal nuclei in addition to the SGCs (arrows) displayed immunoreactivity. In the groups incubated with U0126 or BoNT-A the immunoreactivity had returned to levels found in fresh TG 


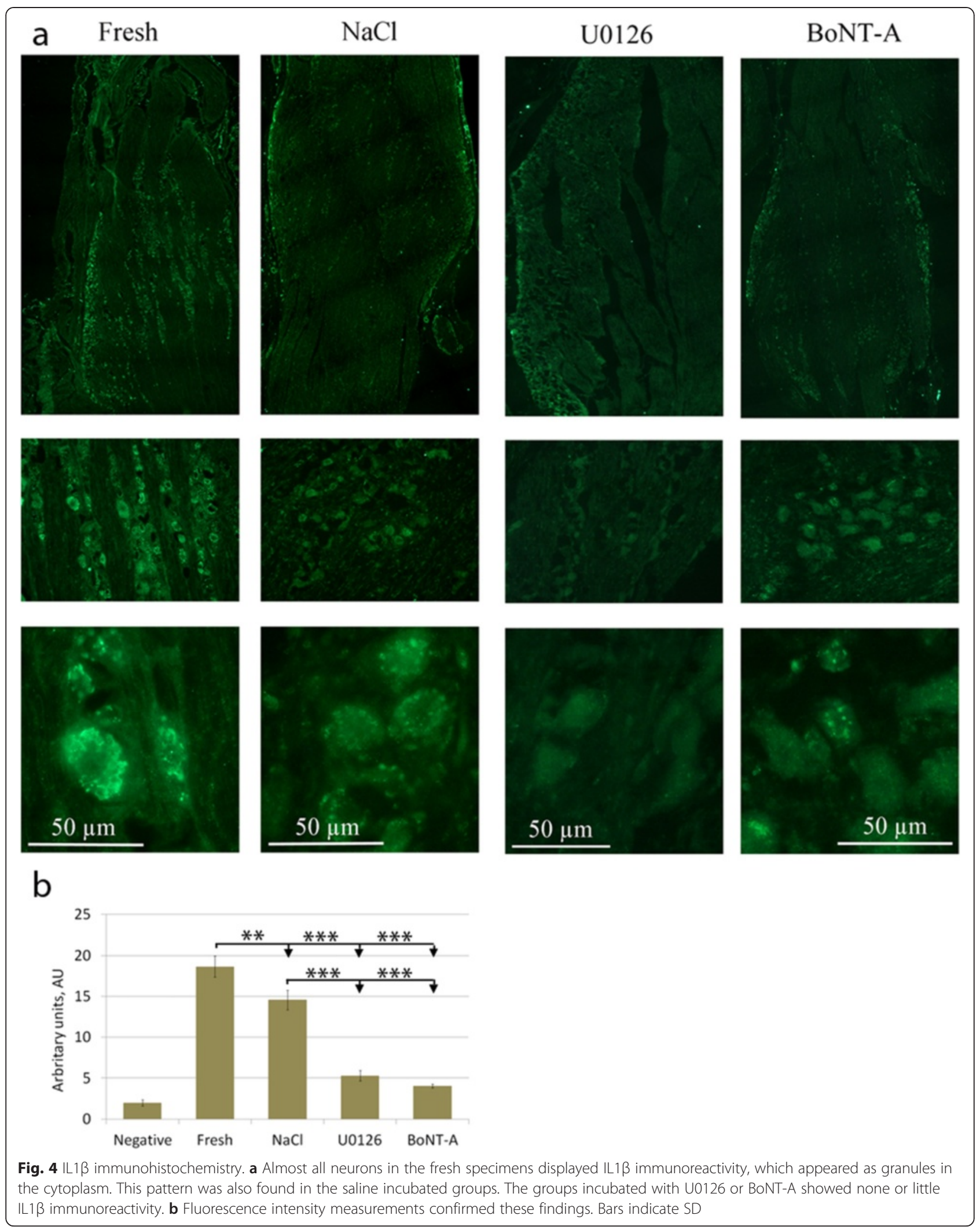



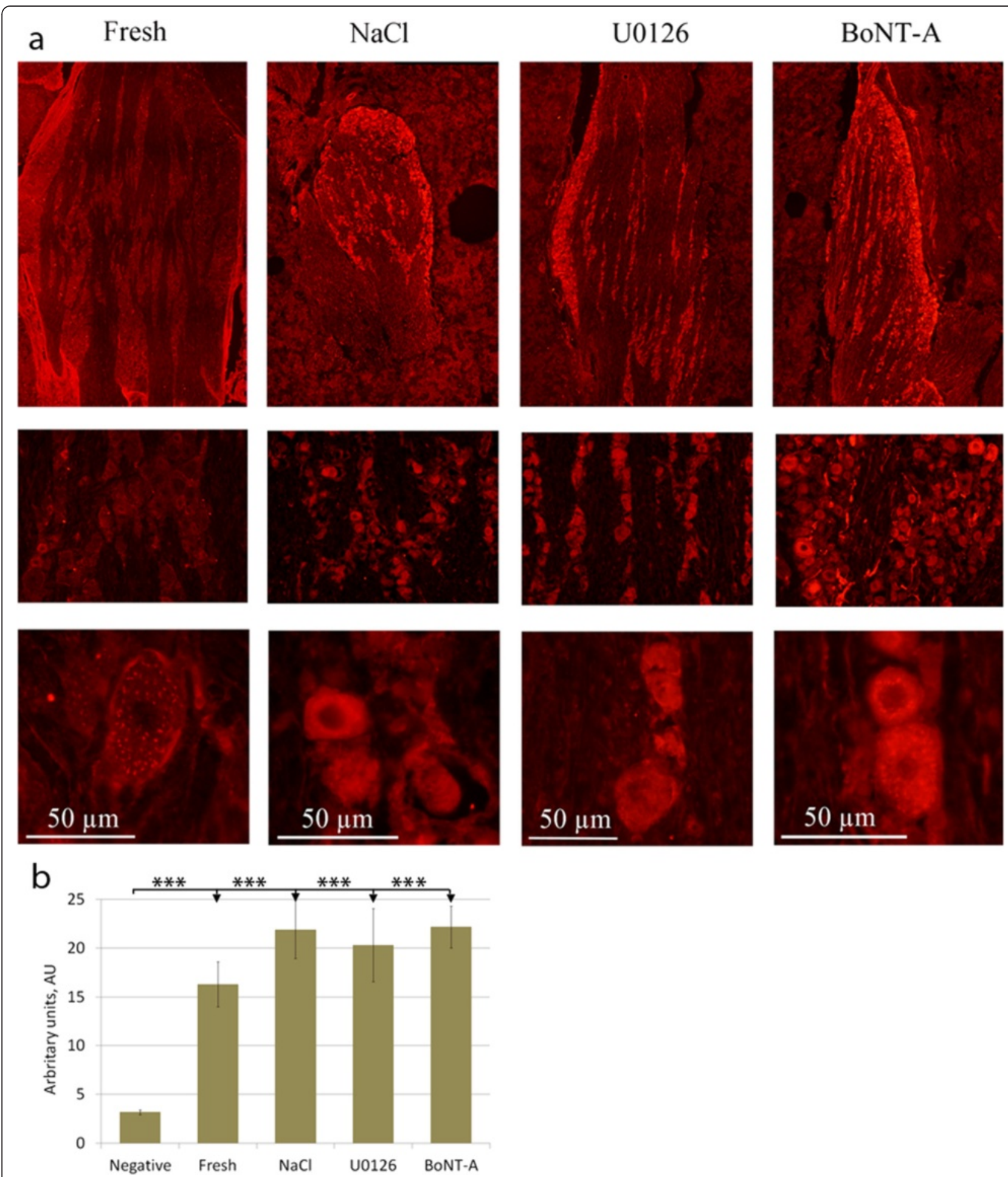

Fig. 5 SNAP-25 immunohistochemistry. a Scattered cytoplasmatic SNAP-25 immunoreactive granules were found in most neurons in the fresh specimens. The intensity of the staining in the incubated groups increased intensively, making it difficult to discern the distribution of immunoreactivity within the neurons. $\mathbf{b}$ Fluorescence intensity measurements confirmed these findindings. Negative column is the same as in SNAP-25 diagram since the same secondary antibody is used in both experiments. Bars indicate SD 


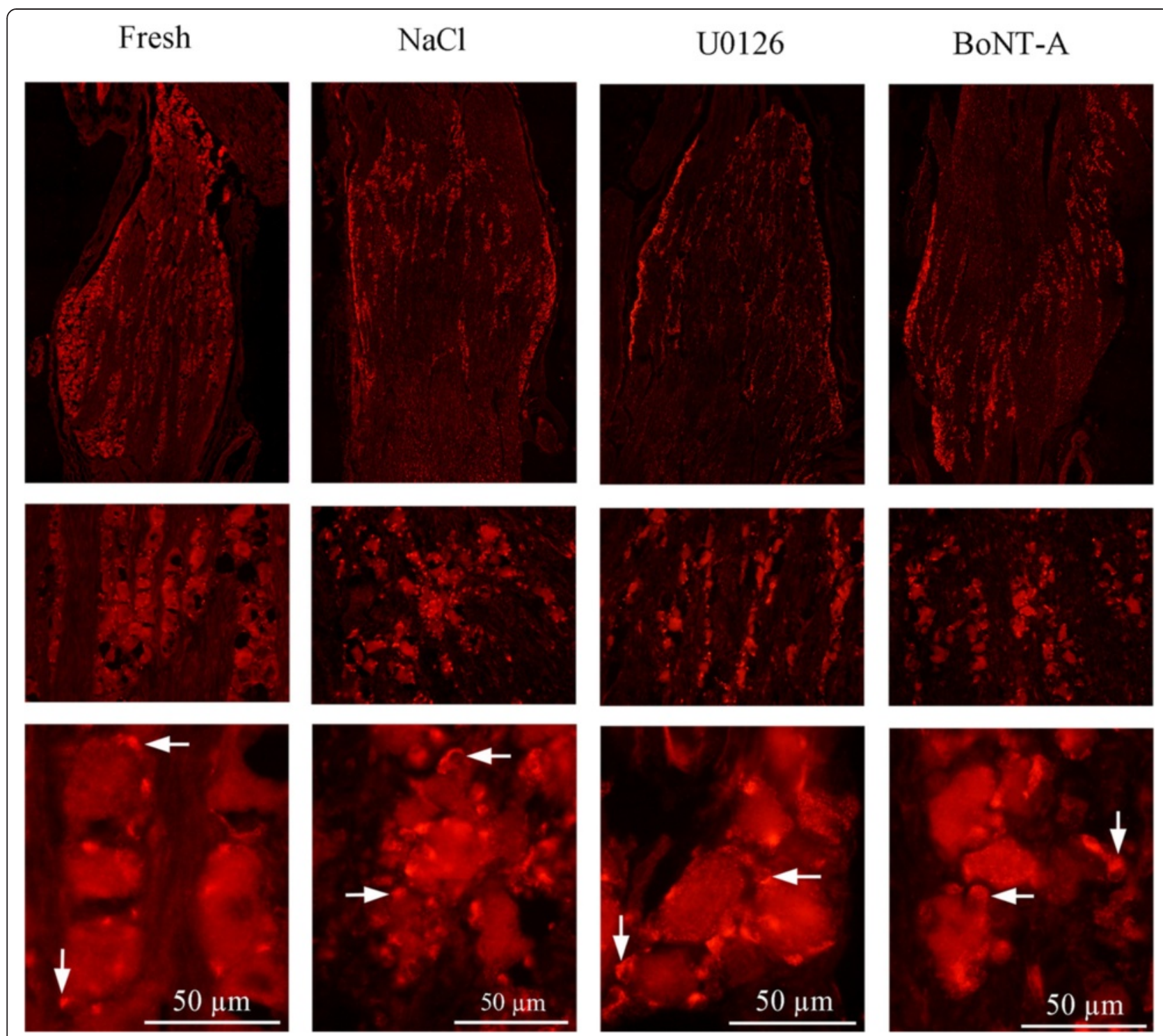

Fig. 6 SV2-A immunohistochemistry. In all groups, including fresh, SV2-A immunoreactivity was confined to the cytoplasm of SGCs (arrows). No difference was detected between the groups, except for the rounded shape of the SGCs appearing after 24 hrs of incubation

\section{U0126 and BoNT-A incubation for $24 \mathrm{hrs}$}

Compared to the fresh specimens, the number of CGRP positive cells was clearly increased in the U0126 group. This was more marked in the BoNT-A treated TG (Fig. 2a). In addition the distribution of the immunoreactivity differed compared to fresh and saline groups; the immunoreactivity was found in the entire cytoplasm in almost all neurons compared to the granular pattern observed in the other groups.

Compared to $\mathrm{NaCl}$ incubated group, iNOS immunoreactivity has returned to levels found in fresh TG (Fig. 3) and IL-1 $\beta$ immunoreactivity has decreased compared to fresh, or saline group (Fig. 4a).

The cytoplasmatic SNAP-25 immunoreactive granules found in neurons in the fresh specimens were visible again (after being hidden in the $\mathrm{NaCl}$ group by the high intensity of the staining), even though the intensity was considerably increased in the U0126 and BoNT-A groups compared to fresh (Fig. 5a). No difference could be seen in the SV2-A immunoreactivity between all the groups examined (Fig. 6).

Fluorescence intensity measurements of CGRP, IL-1 $\beta$ or SNAP-25 were performed on incubated specimens (Figs. 2b, 4b, 5b). The intensity in the neurons was measured and the mean intensity was calculated. Statistically, one-way ANOVA analysis and Bonferroni's multiple comparison test were used. The measurements confirmed the microscopical results and are presented in the illustrations.

Major findings are illustrated schematically in Fig. 7. 


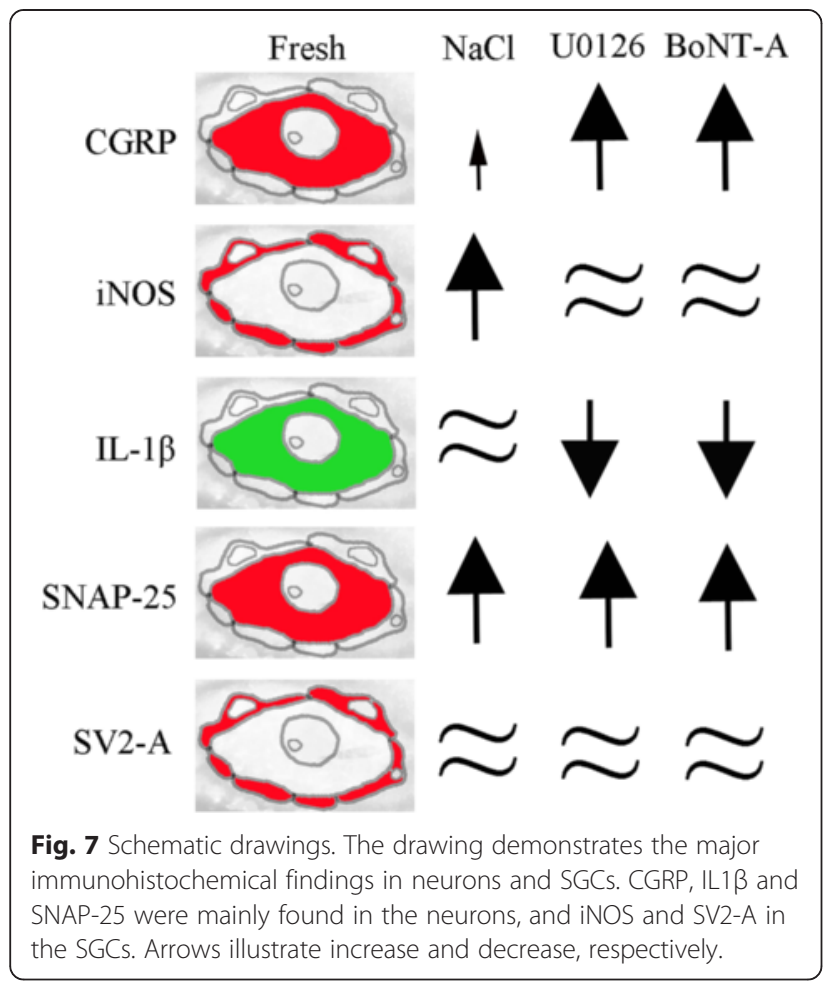

\section{Discussion}

The present study was designed to examine isolated TG neurons and SGCs during different organ culture conditions as a method to illustrate possible expressional alterations of CGRP, iNOS, IL-1 $\beta$, SNAP-25 and SV2-A. The underlying hypothesis is that chronic migraine is associated with inflammation in various parts of the trigeminal system and organ culture is a way to induce an inflammatory response in the TG. The advantage of this method is the study of neurons and SGCs in their normal habitat. We report that the studied molecules were observed in fresh TG with a differential distribution. Interestingly, organ culture co-incubated with the vehicle $\mathrm{NaCl}$ of the TG for 24 hours resulted in enhanced expression of CGRP and SNAP-25 in neurons, and iNOS in SGCs. Co-incubation with U0126 and BoNT-A retained the increased expression of SNAP-25, while it decreased the IL-1 $\beta$ immunoreactivity in neurons. The iNOS expression in SGCs returned to levels observed in fresh specimens. Moreover, we observed no alteration of SV2-A expression in SGCs. However, the overall picture is that both U0126 and BoNT-A have the ability to modify/reduce the expression of certain molecules in the TG.

CGRP was used to provide further in depth information how and if this system are involved using our method. Organ culture results in increased expression of CGRP immunoreactivity in the TG neurons at 48 hours $[14,15]$, but only slightly at 24 hours (present data). Although we did not perform any detailed cell counting in the present study, the localization resembles that described by Eftekhari et al. (2010) [18].

iNOS is expressed by a wide variety of cell types, including neurons and SGCs [19]. We show that iNOS is expressed exclusively in SGCs of the TG. Increased iNOS expression in glial cells is implicated in the etiology of CNS diseases by causing inflammation and cytotoxicity [20, 21]. Inflammatory stimuli, in our case incubation for 24 hours with saline, revealed increased expression of iNOS in SGCs as compared to nonincubated TG. It has been demonstrated that there is increased expression of iNOS in isolated cultured SGCs in response to CGRP and this is mediated by activation of the MAPKs ERK1/2, JNK and p38 [22]. In the present study, we extended the findings to provide evidence that co-incubation with U0126 or BoNT-A show the same expression of iNOS as seen in fresh specimens; this means that these treatments reduced the organ culture induced increase in iNOS expression.

There is IL-1 $1 \beta$ immunoreactivity expressed in many neurons in fresh TG. Co-incubation with saline did not change this pattern of expression, however, the MEK $1 / 2$ inhibitor U0126 and BoNT-A decreased the expression of IL-1 $\beta$. There is increasing evidence that proinflammatory cytokines (IL- $1 \beta$, IL- 6 and TNF- $\alpha$ ) either are synthesized in the central (CNS) or in the peripheral nervous system (PNS) by resident cells, or imported by immune blood cells. These are involved in several pathophysiological functions, including an unexpected impact on synaptic transmission and neuronal excitability. Targeting these cytokines, and related signalling molecules, is considered a novel option for the development of therapies in various CNS or PNS disorders associated with an inflammatory component [23].

BoNTs is known to inhibit the release of excitatory neurotransmitters from both motor and sensory neurons by preventing vesicle fusion to cell membrane [24]. Under pathophysiological conditions, BoNTs prevent neurotransmission from almost every type of neuron suggesting common targets present in the majority of neurons. BoNTs specifically and exclusively attack presynaptic nerve terminals. The fusion of a secretory vesicle with the cell membrane is a highly regulated process with the formation of t-SNARE complex playing a pivotal role. The light chain of BoNTs acts to cleave SNAP-25, which inhibits synaptic exocytosis, and therefore, disables neural transmission [25]. We report that the level of expression of SNAP-25 increased after incubation with BoNT-A, but also after $\mathrm{NaCl}$ and U0126 incubation. This indicates accumulation of SNAP-25 in the cytosol of the neurons, as a suggestion regarding BoNT-A incubated TG, from cleavage of SNAP-25 inhibiting exocytosis. However, other mechanisms may be involved when it comes to TG incubated with $\mathrm{NaCl}$ or U0126. 
Synaptic vesicles (SVs), present in all types of neurons $[26,27]$, are secretory organelles of presynaptic nerve terminals that accumulate high quantities of neurotransmitters and secrete them by fusion with the presynaptic plasma membrane. BoNT-A may enter neurons by binding to synaptic vesicle protein SV2 (isoforms A, B or/and C) [18]. The details of the BoNT-A binding to SV2 has been clarified in hippocampus from knockout mice, demonstrating that those that lacked the isoforms displayed reduced sensitivity to BoNT-A. It was concluded that SV2 acts as the protein receptor for BoNT-A [19]. We showed that the SV2-A isoform is expressed in the SGCs of rat TG, but not in the neurons. This would point towards these cells as a possible site for a putative action of BoNT-A.

The SGCs surround the TG neurons like a pearl necklace, outnumber the neurons in a 10:1 manner, and are joined by gap junctions $[20,28]$. The space between the SGCs and the neurons is $20 \mathrm{~nm}$, which allows for an effective control of the extracellular environment by both neurons and SGCs [20]. The role of the SGCs in the function of the trigeminovascular system is poorly known and has by and large received little attention over the years [20]. In previous work, we observed that the SGCs stored CGRP receptor components (RAMP1, CLR) [18]. In the present study we demonstrate the presence of the inflammation marker iNOS. Vast evidence exists for interaction between the SGCs and the TG neurons. Hence these may be activated via peripheral nociceptors such as those within the dura mater $(20,21)$ and from the temporomandibular joint (TMJ) [23, 29], as well as from central connections in the trigeminal nucleus caudalis (TNC) [13, 30].

The organ culture method has been used in various reports from our laboratory [14-16]. It has been shown that the method could be used to investigate inflammatory events and neuropeptide expression in the TG. In the present study we show that 24 hours of incubation results in condensed nuclear chromatin and compacted cytoplasm in neurons, and enlarged rounded SGCs with highly condensed nuclear chromatin. Moreover, in groups treated with U0126 or BoNT-A, the neurons were often found in a vacuole indicating cell shrinkage and SGC detachment. The impact of these findings in correlation with the expressional alterations of CGRP, iNOS, IL-1 $\beta$, SNAP-25 and SV2-4 can only be speculated upon. Pannese (2010) highlighted the striking morphological changes that SGCs undergo after nerve injury, which includes hypertrophy and formation of bridges with other SGCs, which contain numerous newly formed gap junctions [31]. Obviously, SGCs can sense injury-related changes in the neurons, which might have influenced some of the expressional alterations demonstrated here.
Recently, we asked if a chronic inflammation elicited with injection into the TMJ of CFA [32] or CGRP [33], of CFA on the dura mater (Lukács M, Haanes KA, Majláth Z, Tajti J, Vécsei L, Warfvinge K, Edvinsson L. Exposure of the rat dura mater to inflammatory soup or Complete Freund's Adjuvant induces an inflammatory response in the rat trigeminal ganglion.) might result in altered expression of various markers in the TG. We feel it is of particular importance to maintain the cellular organization because activation of trigeminal neurons leads to changes in adjacent glia that may involve communication through gap junctions and paracrine signaling [17]. The animals were followed for a week and during this time there was a successive increase in inflammation mediators in SGCs and neurons. Interestingly, this enhanced expression could be modified by mitogen-activated protein kinase (MAPK) inhibition in vivo or modified by interacting with the glutamatergic system. In the present method of organ culture we found activation of some of these molecules. The MAPK inhibitor U0126 could modify the expression of CGRP and IL- $\beta$. On the other hand the inhibition of a single molecule such as CGRP receptor telcagepant did not change the expression (data not shown). Interestingly, BoNT-A had the same effect as for U0126.

\section{Conclusion}

We hypothesize that chronic migraine might be associated with some degree of inflammation in the TG that could involve both neurons and SGCs. It is a clinically well recognized that treatment with corticosteroids will reduce the symptoms of chronic migraine; however this remedy is associated with long-term side effects. Understanding the mechanisms involved in the expressional alterations in the trigeminal system may suggest novel ways to modify the changes and indicate novel therapeutics. The results of the present work illustrate one way by which BoNT-A could modify these expressional alterations in the sensory TG.

\section{Abbreviations \\ TG: Trigeminal ganglion; SGC: Satellite glial cells, PBS, Phosphate buffered saline; BSA: Bovine serum albumin; MAPK: Mitogen-activated protein kinases; CGRP: Calcitonin gene-related peptide; U0126: Mitogen activated kinase kinase (EK1/2) inhibitor; BoNT-A: Onabotulinumtoxin type A; iNOS: Inducible nitric oxide synthase; IL-1 $\beta$ : Interleukin $1 \beta$; SNAP-25: Synaptosome-associated protein of $25 \mathrm{kDa}$; SV2-A: Synaptic vesicle protein 2.}

\section{Competing interests}

Dr. Edvinsson reports grants and Botox donation from Allergan during the conduct of the study. In addition, Dr. Edvinsson is consulting on CGRP for Lilly and Teva Pharmaceuticals. JE and KW report no competing interests.

\section{Authors' contribution}

$J E$, KW and LE participated in the design of the study. JE and KW carried out the immunohistochemistry and all three authors participated in the analysis of the results. KW and LE wrote the manuscript. All three authors read and approved the final manuscript. 


\section{Acknowledgments}

Supported by grants from the Swedish Research Council (no 5958) and the Swedish Heart and Lung Foundation.

\section{Disclosures}

The author collaborates have received an unrestricted grant from Allergan and samples of Botox for this preclinical project.

\section{Received: 7 April 2015 Accepted: 11 July 2015}

\section{Published online: 06 August 2015}

\section{References}

1. Lipton RB, Bigal ME, Diamond M, Freitag F, Reed ML, Stewart WF, AMPP Advisory Group (2007) Migraine prevalence, disease burden, and the need for preventive therapy. Neurology 68(5):343-9

2. Akerman S, Holland PR, Goadsby PJ (2011) Diencephalic and brainstem mechanisms in migraine. Nat Rev Neurosci 12(10):570-84

3. Diener HC, Dodick DW, Goadsby PJ, Lipton RB, Olesen J, Silberstein SD (2011) Chronic migraine-classification, characteristics and treatment. Nat Rev Neurol 8(3):162-71

4. Aurora SK, Dodick DW, Turkel CC, DeGryse RE, Silberstein SD, Lipton RB et al. (2010) OnabotulinumtoxinA for treatment of chronic migraine: results from the double-blind, randomized, placebo-controlled phase of the PREEMPT 1 trial. Cephalalgia 30(7):793-803

5. Diener HC, Dodick DW, Aurora SK, Turkel CC, DeGryse RE, Lipton RB et al (2010) OnabotulinumtoxinA for treatment of chronic migraine: results from the double-blind, randomized, placebo-controlled phase of the PREEMPT 2 trial. Cephalalgia 30(7):804-14

6. Dodick DW, Turkel CC, DeGryse RE, Aurora SK, Silberstein SD, Lipton RB et al. (2010) OnabotulinumtoxinA for treatment of chronic migraine: pooled results from the double-blind, randomized, placebo-controlled phases of the PREEMPT clinical program. Headache 50(6):921-36

7. Dolly JO, Lawrence GW, Meng J, Wang J, Ovsepian SV (2009) Neuroexocytosis: botulinum toxins as inhibitory probes and versatile therapeutics. Curr Opin Pharmacol 9(3):326-35

8. Meunier FA, Schiavo G, Molgo J (2002) Botulinum neurotoxins: from paralysis to recovery of functional neuromuscular transmission. J Physiol Paris 96(1-2):105-13

9. Binder WJ, Brin MF, Blitzer A, Schoenrock LD, Pogoda JM (2000) Botulinum toxin type A (BOTOX) for treatment of migraine headaches: an open-label study. Otolaryngol Head Neck Surg 123(6):669-76

10. Dougherty CO, Silberstein SD (2012) OnabotulinumtoxinA in the treatment of migraine headache. Reg Anesth Pain Manag 16:41-6

11. Gazerani P, Pedersen NS, Staahl C, Drewes AM, Arendt-Nielsen L (2009) Subcutaneous Botulinum toxin type $A$ reduces capsaicin-induced trigeminal pain and vasomotor reactions in human skin. Pain 141(1-2):60-9

12. Gazerani P, Au S, Dong X, Kumar U, Arendt-Nielsen L, Cairns BE (2010) Botulinum neurotoxin type A (BoNTA) decreases the mechanical sensitivity of nociceptors and inhibits neurogenic vasodilation in a craniofacial muscle targeted for migraine prophylaxis. Pain 151(3):606-16

13. Burstein R, Zhang X, Levy D, Aoki KR, Brin MF (2014) Selective inhibition of meningeal nociceptors by botulinum neurotoxin type $A$ : therapeutic implications for migraine and other pains. Cephalalgia 34(11):853-69

14. Kuris A, Xu CB, Zhou MF, Tajti J, Uddman R, Edvinsson L (2007) Enhanced expression of CGRP in rat trigeminal ganglion neurons during cell and organ culture. Brain Res 1173:6-13

15. Tajti J, Kuris A, Vécsei $L, X u C B$, Edvinsson L (2011) Organ culture of the trigeminal ganglion induces enhanced expression of calcitonin gene-related peptide via activation of extracellular signal-regulated protein kinase 1/2. Cephalalgia 31(1):95-105

16. Kristiansen KA, Edvinsson L (2010) Neurogenic inflammation: a study of rat trigeminal ganglion. J Headache Pain 11(6):485-95

17. Thalakoti S, Patil W, Damodaram S, Vause CV, Langford LE, Freeman SE et al. (2007) Neuron-glia signaling in trigeminal ganglion: implications for migraine pathology. Headache 47(7):1008-23, discussion 24-5

18. Eftekhari S, Salvatore CA, Calamari A, Kane SA, Tajti J, Edvinsson L (2010) Differential distribution of calcitonin gene-related peptide and its receptor components in the human trigeminal ganglion. Neuroscience 169(2):683-96

19. Fiebich BL, Lieb K, Engels S, Heinrich M (2002) Inhibition of LPS-induced p42/44 MAP kinase activation and iNOS/NO synthesis by parthenolide in rat primary microglial cells. J Neuroimmunol 132(1-2):18-24
20. Murphy S (2000) Production of nitric oxide by glial cells: regulation and potential roles in the CNS. Glia 29(1):1-13

21. Paakkari I, Lindsberg P (1995) Nitric oxide in the central nervous system. Ann Med 27(3):369-77

22. Vause CV, Durham PL (2009) CGRP stimulation of iNOS and NO release from trigeminal ganglion glial cells involves mitogen-activated protein kinase pathways. J Neurochem 110(3):811-21

23. Vezzani A, Viviani B. Neuromodulatory properties of inflammatory cytokines and their impact on neuronal excitability. Neuropharmacology. 2014(96):70-82. Epub 2014 Nov 8. Review.PMID: 25445483

24. Durham PL, Cady R (2011) Insights into the mechanism of onabotulinumtoxinA in chronic migraine. Headache 51(10):1573-7

25. Sutton RB, Fasshauer D, Jahn R, Brunger AT (1998) Crystal structure of a SNARE complex involved in synaptic exocytosis at $2.4 \mathrm{~A}$ resolution. Nature 395(6700):347-53

26. Bajjalieh SM, Scheller RH (1994) Synaptic vesicle proteins and exocytosis. Adv Second Messenger Phosphoprotein Res 29:59-79

27. Janz R, Sudhof TC (1999) SV2C is a synaptic vesicle protein with an unusually restricted localization: anatomy of a synaptic vesicle protein family. Neuroscience 94(4):1279-90

28. Hanani M (2010) Satellite glial cells in sympathetic and parasympathetic ganglia: in search of function. Brain Res Rev 64(2):304-27

29. Durham PL, Garrett FG (2010) Development of functional units within trigeminal ganglia correlates with increased expression of proteins involved in neuron-glia interactions. Neuron Glia Biol 6(3):171-81

30. Dong M, Yeh F, Tepp WH, Dean C, Johnson EA, Janz R et al. (2006) SV2 is the protein receptor for botulinum neurotoxin A. Science 312(5773):592-6

31. Pannese $E$ (2010) The structure of the perineuronal sheath of satellite glial cells (SGCs) in sensory ganglia. Neuron Glia Biol 6(1):3-10

32. Eftekhari S, Warfvinge K, Blixt FW, Edvinsson L (2013) Differentiation of nerve fibers storing CGRP and CGRP receptors in the peripheral trigeminovascular system. J Pain 14(11):1289-303

33. Cady RJ, Glenn JR, Smith KM, Durham PL (2011) Calcitonin gene-related peptide promotes cellular changes in trigeminal neurons and glia implicated in peripheral and central sensitization. Mol Pain 7:94

\section{Submit your manuscript to a SpringerOpen ${ }^{\circ}$ journal and benefit from:}

- Convenient online submission

- Rigorous peer review

- Immediate publication on acceptance

- Open access: articles freely available online

- High visibility within the field

- Retaining the copyright to your article

Submit your next manuscript at $>$ springeropen.com 